\title{
Global well-posedness for the critical Schrödinger-Debye system
}

\author{
Xavier Carvajal and Pedro Gamboa \\ Communicated by Terence Tao, received June 11, 2014.
}

\begin{abstract}
We establish global well-posedness results for the initial value problem associated to the Schrödinger-Debye system in dimension two, for data in $H^{s}\left(\mathbb{R}^{2}\right) \times L^{2}\left(\mathbb{R}^{2}\right), 2 / 3<s \leq 1$ and for data in $H^{1}\left(\mathbb{R}^{2}\right) \times H^{1}\left(\mathbb{R}^{2}\right)$.
\end{abstract}

\section{Contents}

1. Introduction

2. notation and basic properties

3. Global Well-posedness in $H^{1}\left(\mathbb{R}^{2}\right) \times H^{1}\left(\mathbb{R}^{2}\right)$

4. Global well-posedness in $H^{s}\left(\mathbb{R}^{2}\right) \times L^{2}\left(\mathbb{R}^{2}\right), 2 / 3<s \leq 1 \quad 259$

References

Bibliografia

\section{Introduction}

We consider the initial value problem (IVP) for the Schrödinger-Debye system

$$
\begin{cases}i u_{t}+\frac{1}{2} \Delta u=u v, \quad t>0, & x \in \mathbb{R}^{n}, \\ \mu v_{t}+v=\lambda|u|^{2}, \quad \mu>0, & \lambda= \pm 1, \\ u(0)=u_{0}, \quad v(0)=v_{0}, & \end{cases}
$$

where $u=u(x, t)$ is a complex-valued function, $v=v(x, t)$ is a real-valued function and $\Delta$ is the Laplacian operator in the spatial variable. This model describes the propagation of an electromagnetic wave through a non-resonant medium whose material response time is relevant. See Newel and Moloney $[\mathbf{1 0}]$ for a more complete

1991 Mathematics Subject Classification. 35A01, 35Q53.

Key words and phrases. Schrödinger-Debye system, Global well-posedness, A priori estimates. 
discussion of this model. In the absence of the delay $(\mu=0)$, the system (1.1) reduces to the cubic nonlinear Schrödinger equation (NLS)

$$
i u_{t}+\frac{1}{2} \Delta u=\lambda u|u|^{2},
$$

which is focusing or defocusing for $\lambda=-1$ and 1, respectively. Similarly, the sign of the parameter $\lambda$ provides an analogous classification for (1.1). For sufficiently regular data, the mass of the solution $u$ of the system (1.1) is invariant. More precisely,

$$
\int_{\mathbb{R}^{n}}|u(x, t)|^{2} d x=\int_{\mathbb{R}^{n}}\left|u_{0}(x)\right|^{2} d x .
$$

The system (1.1) has the following pseudo-Hamiltonian structure

$$
\frac{d}{d t} E(u(t), v(t))=2 \lambda \mu \int_{\mathbb{R}^{n}}\left|v_{t}\right|^{2} d x,
$$

where

$$
\begin{aligned}
E(u(t), v(t)) & :=E(u, v)=\int_{\mathbb{R}^{n}}\left(|\nabla u|^{2}+2 v|u|^{2}-\lambda v^{2}\right) d x \\
& =\int_{\mathbb{R}^{n}}\left(|\nabla u|^{2}+\lambda|u|^{4}-\lambda \mu^{2}\left|v_{t}\right|^{2}\right) d x .
\end{aligned}
$$

Also, the system (1.1) is equivalent to the following integral form

$$
u(t)=S(t) u_{0}+i \int_{0}^{t} S(t-\tau) u(\tau) v(\tau) d \tau
$$

and

$$
v(t)=e^{-\frac{t}{\mu}} v_{0}+\frac{\lambda}{\mu} \int_{0}^{t} e^{-\frac{(t-\tau)}{\mu}}|u(\tau)|^{2} d \tau,
$$

where $S(t)=e^{i t \Delta / 2}$ is the unitary Schrödinger group.

The well-posedness of the system (1.1) has been studied by different authors. In 2000, Bidégaray ([4] and [5]) studied the local well-posedness (LWP) of the system (1.1) in dimensions $n=1,2,3$ and for data in $H^{s}\left(\mathbb{R}^{n}\right) \times H^{s}\left(\mathbb{R}^{n}\right), s>n / 2, s=0$ and $s=1$. In 2004, Corcho and Linares ([7]) obtained the best LWP result in dimension one. Later, in 2009, Corcho and Matheus ([8]) obtained a refined LWP and global-well posedness (GWP) result in the framework of Bourgain spaces, also in dimension one. Recently, Corcho, Oliveira e Silva (see [9]), also in the framework of Bourgain spaces $X_{s, b}$ (see definition in Section 2), obtained the following LWP result in dimensions two and three.

Theorem 1.1. Let $n=2,3$. For any $\left(u_{0}, v_{0}\right) \in H^{s_{1}}\left(\mathbb{R}^{n}\right) \times H^{s_{2}}\left(\mathbb{R}^{n}\right)$, with $s_{1}$ and $s_{2}$ satisfying the conditions

$$
\max \left\{0, s_{1}-1\right\} \leq s_{2} \leq \min \left\{2 s_{1}, s_{1}+1\right\},
$$

there exists a positive time

$$
T=T\left(\left\|u_{0}\right\|_{H^{s_{1}}},\left\|v_{0}\right\|_{H^{s_{2}}}\right)
$$

and a unique solution $(u(t), v(t))$ of the IVP (1.1) on the time interval $[0, T]$, such that

$$
\text { (i) }\left(\psi_{T} u(t), \psi_{T} v(t)\right) \in X_{s_{1}, b} \times H_{s_{2}, c} \text {; }
$$


(ii) $(u, v) \in C\left([0, T] ; H^{s_{1}}\left(\mathbb{R}^{n}\right) \times H^{s_{2}}\left(\mathbb{R}^{n}\right)\right)$

for suitable $b$ and $c$ close to $1 / 2+\left(\psi_{T}\right.$ denotes, as usual, a cutoff function for the time interval $[0, T])$. Moreover, the map $\left(u_{0}, v_{0}\right) \rightarrow(u(t), v(t))$ is locally Lipschitz from $H^{s_{1}}\left(\mathbb{R}^{n}\right) \times H^{s_{2}}\left(\mathbb{R}^{n}\right)$ into $C\left([0, T] ; H^{s_{1}}\left(\mathbb{R}^{n}\right) \times H^{s_{2}}\left(\mathbb{R}^{n}\right)\right)$.

In the following graphic we resume the LWP in the above theorem:

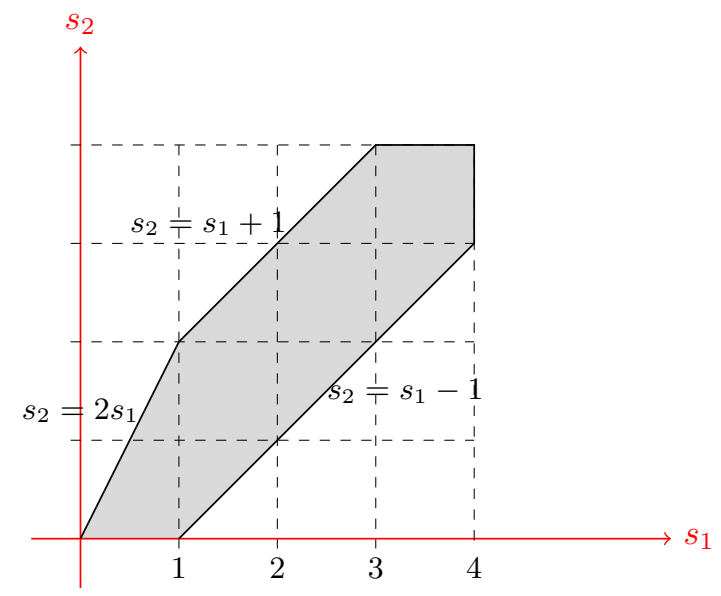

The shaded region represents the region where the LWP result exists. They also proved the following GWP result in dimension two.

Theorem 1.2. Let $\left(u_{0}, v_{0}\right) \in H^{1}\left(\mathbb{R}^{2}\right) \times L^{2}\left(\mathbb{R}^{2}\right)$. Then, for all $T>0$, there exists a unique solution

$$
(u, v) \in C\left([0, T] ; H^{1}\left(\mathbb{R}^{2}\right) \times L^{2}\left(\mathbb{R}^{2}\right)\right)
$$

to the initial value problem (1.1), such that

$$
\|\nabla u(., t)\|_{L_{x}^{2}}^{2}+\|v(., t)\|_{L_{x}^{2}}^{2} \leq \alpha_{0} e^{\alpha_{1} T}, \quad t \in[0, T], \quad T>0,
$$

where $\alpha_{0}=\alpha_{0}\left(E\left(u_{0}, v_{0}\right),\left\|v_{0}\right\|_{L_{x}^{2}}^{2},\left\|u_{0}\right\|_{L_{x}^{2}}^{2}\right)$ and $\alpha_{1}=\alpha_{1}\left(\left\|u_{0}\right\|_{L_{x}^{2}}^{2}\right)$.

In this work we prove the following GWP result in the space $H^{1}\left(\mathbb{R}^{2}\right) \times H^{1}\left(\mathbb{R}^{2}\right)$.

Theorem 1.3. Let $\left(u_{0}, v_{0}\right) \in H^{1}\left(\mathbb{R}^{2}\right) \times H^{1}\left(\mathbb{R}^{2}\right)$. Then, for all $T>0$, there exists a unique solution

$$
(u, v) \in C\left([0, T] ; H^{1}\left(\mathbb{R}^{2}\right) \times H^{1}\left(\mathbb{R}^{2}\right)\right)
$$

to the initial value problem (1.1).

This solves a problem left open in Corcho, Oliveira and Silva [9]; see Remark 4.3 therein. The proof of this theorem is simple; it uses basic properties of the unitary Schrödinger group and the Gronwell inequality.

Next we prove a result of GWP (small data) below energy space for the Schrödinger solution $u$. We will use the method of Bourgain ([3]) on high and low frequencies together with the framework of dispersive Sobolev spaces.

THeOREM 1.4. Let $\left(u_{0}, v_{0}\right) \in H^{s}\left(\mathbb{R}^{2}\right) \times L^{2}\left(\mathbb{R}^{2}\right)$, with $2 / 3<s \leq 1$, such that

$$
2 c_{0}^{2}\left\|u_{0}\right\|_{L_{x}^{2}}<1
$$


where $c_{0}$ is the best constant in the Gagliardo-Nirenberg inequality

$$
\|u\|_{L_{x}^{4}\left(\mathbb{R}^{2}\right)} \leq c_{0}\|u\|_{L_{x}^{2}\left(\mathbb{R}^{2}\right)}^{1 / 2}\|u\|_{H_{x}^{1}\left(\mathbb{R}^{2}\right)}^{1 / 2} .
$$

Then, for all $T>0$, there exists a unique solution

$$
(u, v) \in C\left([0, T] ; H^{s}\left(\mathbb{R}^{2}\right) \times L^{2}\left(\mathbb{R}^{2}\right)\right)
$$

to the initial value problem (1.1) with $\lambda=-1$.

The difficulty in the proof of these theorems lies in the absence of conserved quantities in the energy space.

\section{2. notation and basic properties}

Let $Y_{x}$ be a normed space on $\mathbb{R}^{n}$. We denote by $L_{t}^{p} Y_{x}\left(J \times \mathbb{R}^{n}\right):=L_{t}^{p}\left(J ; Y_{x}\left(\mathbb{R}^{n}\right)\right)$, where $J \subseteq \mathbb{R}$ is an interval, the completion of the space of Schwartz functions $f(x, t)$, with the norm

$$
\|f\|_{L_{t}^{p} Y_{x}\left(J \times \mathbb{R}^{n}\right)}=\left(\int_{J}\|f(t)\|_{Y_{x}}^{p} d t\right)^{1 / 2} .
$$

When $\mathbb{R}^{n}$ and $J$ are implicit, we denote this norm by $\|f\|_{L_{t}^{p} Y_{x}}$.

Let $\mathrm{h}$ be a continuous function on $\mathbb{R}$. We define the space $X_{s, b}$ by

$$
X_{s, b}=X_{s, b}^{h}\left(\mathbb{R}^{n} \times \mathbb{R}\right):={\overline{S_{x, t}\left(\mathbb{R}^{n} \times \mathbb{R}\right.}}^{\|\cdot\|_{X_{s, b}},}
$$

where $S_{x, t}$ is the Schwartz space, with norm

$$
\|u\|_{X_{s, b}^{h(\xi)}}:=\left\|\langle\xi\rangle^{s}\langle\tau-h(\xi)\rangle^{b} \widetilde{u}(\xi, \tau)\right\|_{L_{\tau}^{2} L_{x}^{2}}
$$

with the notation $\langle\cdot\rangle:=\left\{1+|\cdot|^{2}\right\}^{1 / 2} \sim 1+|\cdot|$, recalling that the Fourier transform (space-time) is given by

$$
\widetilde{u}(\xi, \tau)=\int_{\mathbb{R}} \int_{\mathbb{R}^{n}} e^{-i(x \cdot \xi+t \tau)} u(x, t) d x d t .
$$

The space $X_{s, b}$ is called Bourgain space or dispersive Sobolev space. Similarly we define the space $H_{l, c}$ with norm

$$
\|u\|_{H_{l, c}}:=\left\|\langle\xi\rangle^{l}\langle\tau\rangle^{c} \widetilde{u}(\xi, \tau)\right\|_{L_{\tau}^{2} L_{x}^{2}} .
$$

Let $I \subset \mathbb{R}$ be any interval of time. We define

with norm

$$
X_{s, b}\left(\mathbb{R}^{n} \times I\right):=\left\{u ; u=\left.v\right|_{\mathbb{R}^{n} \times I}, v \in X_{s, b}^{h}\left(\mathbb{R}^{n} \times \mathbb{R}\right)\right\}
$$

$$
\|u\|_{X_{s, b}\left(\mathbb{R}^{n} \times I\right)}:=\inf \left\{\|v\|_{X_{s, b}} ;\left.v\right|_{\mathbb{R}^{n} \times I}=u\right\} .
$$

We have that

$$
X_{s, 0}=L_{t}^{2} H_{x}^{s} \quad \text { and } \quad\left(X_{s, b}^{h(\xi)}\right)^{\prime}=X_{-s,-b}^{-h(-\xi)}
$$

and moreover,

$$
X_{s, b} \hookrightarrow C\left(\mathbb{R} ; H^{s}\left(\mathbb{R}^{n}\right)\right) \quad \text { and } \quad H_{l, c} \hookrightarrow C\left(\mathbb{R} ; H^{l}\left(\mathbb{R}^{n}\right)\right),
$$

for any $b, c>1 / 2$ and $s, l \in \mathbb{R}$.

Proposition 2.1. We have the following known embedding 
(1) if $2 \leq p<\infty$ and $b \geq \frac{1}{2}-\frac{1}{p}$ then $\|u\|_{L_{t}^{p} H_{x}^{s}} \leq c\|u\|_{X_{s, b}}$,

(2) if $2 \leq p, q<\infty$ and $b \geq \frac{1}{2}-\frac{1}{p}$ and $s \geq \frac{n}{2}-\frac{1}{q} \quad$ then $\|u\|_{L_{t}^{p} L_{x}^{q}} \leq$ $c\|u\|_{X_{s, b}}$,

(3) if $1<p \leq 2$ and $b \leq \frac{1}{2}-\frac{1}{p}$ then $\|u\|_{X_{s, b}} \leq c\|u\|_{L_{t}^{p} H_{x}^{s}}$,

$$
\begin{aligned}
& \quad\left\|\mathcal{D}_{x}^{1 / 2^{-}}\left(u_{1} u_{2}\right)\right\|_{L_{t}^{2} L_{x}^{2}} \leq c\left\|u_{1}\right\|_{X_{1 / 2,1 / 2}+}\left\|u_{2}\right\|_{X_{0,1 / 2^{-}}}, \\
& \text {and } \\
& \qquad\left\|\mathcal{D}_{x}^{1 / 2^{+}}\left(u_{1} u_{2}\right)\right\|_{L_{t}^{2} L_{x}^{2}} \leq c\left\|u_{1}\right\|_{X_{1 / 2+, 1 / 2}+}\left\|u_{2}\right\|_{X_{0^{+}, 1^{+} 2^{+}}}, \\
& \text {where } \widehat{\left(\mathcal{D}_{x}^{s} f\right)}(\xi)=\langle\xi\rangle^{s} \widehat{f}(\xi) \text {. }
\end{aligned}
$$

Proof. For the items (1), (2) and (3) see [11] and for the item (4) see [3].

We define an operator $\mathcal{L}$ by $\widehat{\mathcal{L} u}(\xi)=-i h(\xi) \widehat{u}(\xi)$.

Proposition 2.2. Let $f \in H_{x}^{s}\left(\mathbb{R}^{d}\right)$ for some $s \in \mathbb{R}$ and for some polynomial $h: \mathbb{R}^{d} \rightarrow \mathbb{R}$. Then

i) For any Schwartz time cutoff $\eta \in S_{x}(\mathbb{R})$, we have

$$
\left\|\eta(t) e^{t \mathcal{L}} f\right\|_{X_{s, b}^{h(\xi)}}\left(\mathbb{R} \times \mathbb{R}^{d}\right) \lesssim \eta, b\|f\|_{H_{x}^{s}\left(\mathbb{R}^{d}\right)} .
$$

ii) Let $Y$ be a Banach space of functions on $\mathbb{R} \times \mathbb{R}^{d}$ with the property that

$$
\left\|e^{i t \tau_{0}} e^{t \mathcal{L}} f\right\|_{Y} \lesssim\|f\|_{H_{x}^{s}\left(\mathbb{R}^{d}\right)},
$$

for all $f \in H_{x}^{s}\left(\mathbb{R}^{d}\right)$. Then we have the embedding

$$
\|u\|_{Y} \lesssim_{b}\|u\|_{X_{s, b}^{h(\xi)}\left(\mathbb{R} \times \mathbb{R}^{d}\right)}
$$

for $b>1 / 2$.

Proof. See $[\mathbf{1 1}]$.

Now define the function $\vartheta: \mathbb{R} \rightarrow \mathbb{R}$ by

$$
\vartheta(t):= \begin{cases}1 & |t|<1 \\ 0 & |t| \geq 2\end{cases}
$$

and $\vartheta \in C_{0}^{\infty}(\mathbb{R})$. Moreover, define $\vartheta_{T}(t):=\vartheta\left(\frac{t}{T}\right)$.

Consider the IVP

$$
\left\{\begin{array}{l}
u_{t}-\mathcal{L} u=F \\
u(0)=u_{0}
\end{array}\right.
$$

The solution of this IVP is given by

$$
u(t)=e^{i t \mathcal{L}} u_{0}+\int_{0}^{t} e^{i(t-s) \mathcal{L}} F(s) d s .
$$


THEOREM 2.1. Let $u$ be the solution of the integral equation (2.3). If $s \in \mathbb{R}$ and $b>\frac{1}{2}$, then

$$
\left\|\vartheta_{T} u\right\|_{X_{s, b}^{h(\xi)}} \leq C\left\|u_{0}\right\|_{H_{x}^{s}}+C\|F\|_{X_{s, b-1}^{h(\xi)}} .
$$

ProOF. See [11].

We establish the properties of the group $\left\{e^{i t \Delta}\right\}_{t=-\infty}^{\infty}$ in the $L^{p}\left(\mathbb{R}^{n}\right)-$ space.

Proposition 2.3. If $t \neq 0, \frac{1}{p}+\frac{1}{p^{\prime}}=1$ and $p^{\prime} \in[1,2]$, then we have $e^{i t \Delta}$ : $L_{x}^{p^{\prime}}\left(\mathbb{R}^{n}\right) \rightarrow L_{x}^{p}\left(\mathbb{R}^{n}\right)$ is continuous and

$$
\left\|e^{i t \Delta} f\right\|_{L_{x}^{p}\left(\mathbb{R}^{n}\right)} \leq c|t|^{-n / 2\left(1 / p^{\prime}-1 / p\right)}\|f\|_{L_{x}^{p^{\prime}}\left(\mathbb{R}^{n}\right)} .
$$

For a proof of this proposition we refer to $[\mathbf{1 1}]$.

We proceed with the notion of admissible pair.

DEFINITION 2.1. We say that the exponent pair $(q, r)$ is admissible if

$$
\frac{2}{q}=n\left(\frac{1}{2}-\frac{1}{r}\right)
$$

where

$$
\begin{array}{ll}
\text { - } 2 \leq r \leq \frac{2 n}{n-2} & \text { if } \quad n>2 \text {, } \\
\text { - } 2 \leq r<\infty & \text { if } \quad n=2 \text {, } \\
\text { - } 2 \leq r \leq \infty & \text { if } \quad n=1 \text {. }
\end{array}
$$

Proposition 2.4 (Strichartz estimates). If $n \geq 1, s \in \mathbb{R},\left(q_{1}, r_{1}\right)$ and $\left(q_{2}, r_{2}\right)$ are admissible and $\frac{1}{q_{2}}+\frac{1}{q_{2}^{\prime}}=1, \frac{1}{r_{2}}+\frac{1}{r_{2}^{\prime}}=1$, then we have the homogeneous Strichartz estimate

$$
\left\|e^{i t \Delta} u_{0}\right\|_{L_{t}^{q_{1}} L_{x}^{r_{1}}\left(\mathbb{R} \times \mathbb{R}^{n}\right)} \leq C(n, q, r)\left\|u_{0}\right\|_{L_{x}^{2}\left(\mathbb{R}^{n}\right)},
$$

the dual homogeneous Strichartz estimate

$$
\left\|\int_{0}^{t} e^{i s \Delta} F(s) d s\right\|_{L_{x}^{2}\left(\mathbb{R}^{n}\right)} \leq C\left(n, q_{2}, r_{2}\right)\|F\|_{L_{t}^{q_{2}^{\prime}} L_{x}^{r_{2}^{\prime}}\left(\mathbb{R} \times \mathbb{R}^{n}\right)},
$$

and the inhomogeneous Strichartz estimate

$$
\left\|\int_{0}^{t} e^{i(t-s) \Delta} F(s) d s\right\|_{L_{t}^{q} L_{x}^{r}\left(\mathbb{R} \times \mathbb{R}^{n}\right)} \leq C\left(n, q_{1}, r_{1}, q_{2}, r_{2}\right)\|F\|_{L_{t}^{q_{2}^{\prime}} L_{x}^{r_{2}^{\prime}}\left(\mathbb{R} \times \mathbb{R}^{n}\right)} .
$$

Proof. See [11].

\section{Global Well-posedness in $H^{1}\left(\mathbb{R}^{2}\right) \times H^{1}\left(\mathbb{R}^{2}\right)$}

In this paper we give a negative answer to the question of the existence of blow-up solutions for the initial data in $H^{1}\left(\mathbb{R}^{2}\right) \times H^{1}\left(\mathbb{R}^{2}\right)$ in Corcho, Oliveira and Silva [9]; see Remark 4.3 therein.

In order to prove a global theory in $H^{1}\left(\mathbb{R}^{2}\right) \times H^{1}\left(\mathbb{R}^{2}\right)$ we need an estimate $\nabla v($.$) . To achieve this, we apply the gradient in the equation (1.6) and we obtain$

$$
\nabla v(t)=\nabla v_{0} e^{-\frac{t}{\mu}}+\frac{\lambda}{\mu} \int_{0}^{t} e^{-(t-\tau) / \mu} \nabla|u(\tau)|^{2} d \tau
$$


Observe that,

$$
\nabla|u|^{2}=\nabla\{u \cdot \bar{u}\}=2 \operatorname{Re}(u \cdot \overline{\nabla u}) .
$$

Replacing this expression in (3.1), we get

$$
\|\nabla v(t)\|_{L_{x}^{2}} \leq\left\|\nabla v_{0}\right\|_{L_{x}^{2}}+2\left|\frac{\lambda}{\mu}\right| \int_{0}^{t} e^{-(t-\tau) / \mu}|| u(\tau) . \nabla u(\tau) \|_{L_{x}^{2}} d \tau .
$$

Therefore

$$
\begin{aligned}
\|\nabla v(t)\|_{L_{x}^{2}} & \leq\left\|\nabla v_{0}\right\|_{L_{x}^{2}}+2\left|\frac{\lambda}{\mu}\right| \int_{0}^{t} e^{-(t-\tau) / \mu}\|u(\tau)\|_{L_{x}^{4}}\|\nabla u(\tau)\|_{L_{x}^{4}} d \tau \\
& \leq\left\|\nabla v_{0}\right\|_{L_{x}^{2}}+\sqrt{\frac{2}{\mu}}\|u(t)\|_{L_{t}^{4} L_{x}^{4}}\|\nabla u(t)\|_{L_{t}^{4} L_{x}^{4}} .
\end{aligned}
$$

Moreover, applying the gradient in the equation (1.5), we have

$$
\nabla u=S(t) \nabla u_{0}+i\left(I_{1}+I_{2}\right),
$$

where

$$
I_{1}=\int_{0}^{t} S(t-\tau) \nabla u(\tau) \cdot v(\tau) d \tau, \quad I_{2}=\int_{0}^{t} S(t-\tau) u(\tau) \cdot \nabla v(\tau) d \tau
$$

Since $(4,4)$ is an admissible pair, we conclude that

$$
\|\nabla u\|_{L_{t}^{4} L_{x}^{4}} \leq c\left\|\nabla u_{0}\right\|_{L_{x}^{2}}+\left\|I_{1}\right\|_{L_{t}^{4} L_{x}^{4}}+\left\|I_{2}\right\|_{L_{t}^{4} L_{x}^{4}},
$$

and using the inhomogeneous Strichartz estimate (2.7), we have

$$
\left\|I_{1}\right\|_{L_{t}^{4} L_{x}^{4}} \leq\left. c\|\nabla u(\tau) \cdot v(\tau)\|\right|_{L_{t}^{4 / 3} L_{x}^{4 / 3}} \leq c\|\nabla u\|_{L_{t}^{2} L_{x}^{2}}\|v\|_{L_{t}^{4} L_{x}^{4}} .
$$

By immersion

$$
\|v\|_{L_{x}^{4}} \leq c_{0}\|v\|_{L_{x}^{2}}^{1 / 2}\|\nabla v\|_{L_{x}^{2}}^{1 / 2}
$$

and from (3.6) and (3.7), we have

$$
\begin{aligned}
\left\|I_{1}\right\| L_{t}^{4} L_{x}^{4} & \leq c\|\nabla u\|_{L_{t}^{2} L_{x}^{2}}\left(\int_{0}^{t}\|v\|_{L_{x}^{2}}^{2}\|\nabla v\|_{L_{x}^{2}}^{2} d \tau\right)^{1 / 4} \\
& \leq c\|\nabla u\|_{L_{t}^{2} L_{x}^{2}}\|v\|_{L_{t}^{\infty} L_{x}^{2}}^{1 / 2}\left(\int_{0}^{t}\|\nabla v\|_{L_{x}^{2}}^{2} d \tau\right)^{1 / 4} \\
& \leq c\|\nabla u\|_{L_{t}^{2} L_{x}^{2}}^{2}\|v\|_{L_{t}^{\infty} L_{x}^{2}}+\left(\int_{0}^{t}\|\nabla v\|_{L_{x}^{2}}^{2} d \tau\right)^{1 / 2}
\end{aligned}
$$

Now we estimate $I_{2}$ :

$$
\begin{aligned}
\left\|I_{2}\right\|_{L_{t}^{4} L_{x}^{4}} & \leq\|u(\tau) \cdot \nabla v(\tau)\|_{L_{t}^{4 / 3} L_{x}^{4 / 3}} \\
& \leq c\left\|u_{0}\right\|_{L_{x}^{2}}^{1 / 2}\|\nabla u\|_{L_{t}^{2} L_{x}^{2}}^{1 / 2}\left(\int_{0}^{t}\|\nabla v\|_{L_{x}^{2}}^{2} d \tau\right)^{1 / 2}
\end{aligned}
$$


Here we used (3.7) and the conserved quantity (1.2). Replacing (3.9) and (3.8) in (3.5), we get

$$
\begin{gathered}
\|\nabla u\|_{L_{t}^{4} L_{x}^{4}} \leq c\left\|\nabla u_{0}\right\|_{L_{x}^{2}}+c\|\nabla u\|_{L_{t}^{2} L_{x}^{2}}^{2}\|v\|_{L_{t}^{\infty} L_{x}^{2}}+\left(\int_{0}^{t}\|\nabla v\|_{L_{x}^{2}}^{2} d \tau\right)^{1 / 2} \\
+c\left\|u_{0}\right\|_{L_{x}^{2}}^{1 / 2}\|\nabla u\|_{L_{t}^{2} L_{x}^{2}}^{1 / 2}\left(\int_{0}^{t}\|\nabla v\|_{L_{x}^{2}}^{2} d \tau\right)^{1 / 2} .
\end{gathered}
$$

Using (3.7), it follows that

$$
\|u\|_{L_{t}^{4} L_{x}^{4}} \leq T_{\max }^{1 / 4}\|u\|_{L_{t}^{\infty} L_{x}^{4}} \leq c_{0} T_{\max }^{1 / 4}\left\|u_{0}\right\|_{L_{x}^{2}}^{1 / 2}\|\nabla u\|_{L_{t}^{\infty} L_{x}^{2}}^{1 / 2} .
$$

Combining (3.3), (3.10) and (3.11), we can show that

$$
\begin{aligned}
& \|\nabla v(t)\|_{L_{x}^{2}} \leq\left\|\nabla v_{0}\right\|_{L_{x}^{2}}+\mathcal{K}_{T}\|\nabla u(t)\|_{L_{t}^{4} L_{x}^{4}} \\
& \leq\left\|\nabla v_{0}\right\|_{L_{x}^{2}}+\mathcal{K}_{T}\left\{\left\|\nabla u_{0}\right\|_{L_{x}^{2}}+\|\nabla u\|_{L_{t}^{2} L_{x}^{2}}^{2}\|v\|_{L_{t}^{\infty} L_{x}^{2}}+\left(\int_{0}^{t}\|\nabla v\|_{L_{x}^{2}}^{2} d \tau\right)^{1 / 2}\right\}, \\
& +\mathcal{K}_{T}\left\|u_{0}\right\|_{L_{x}^{2}}^{1 / 2}\|\nabla u\|_{L_{T}^{\infty} L_{x}^{2}}^{1 / 2}\left(\int_{0}^{t}\|\nabla v\|_{L_{x}^{2}}^{2} d \tau\right)^{1 / 2}
\end{aligned}
$$

where

$$
\mathcal{K}_{T}=c_{0} \sqrt{\frac{2}{\mu}} T_{\text {max }}^{1 / 4}\left\|u_{0}\right\|_{L_{x}^{2}}^{1 / 2}\|\nabla u\|_{L_{t}^{\infty} L_{x}^{2}}^{1 / 2} .
$$

Observe that

$$
\|\nabla v(t)\|_{L_{x}^{2}} \leq \Phi+G(T)\left(\int_{0}^{t}\|\nabla v(\tau)\|_{L_{x}^{2}}^{2} d \tau\right)^{1 / 2}
$$

where

$$
\begin{gathered}
G(T)=\mathcal{K}_{T}\left(1+\left\|u_{0}\right\|_{L_{x}^{2}}^{1 / 2}\|\nabla u\|_{L_{T}^{\infty} L_{x}^{2}}^{1 / 2}\right) \\
\Phi=\left\|\nabla v_{0}\right\|_{L_{x}^{2}}+\mathcal{K}_{T}\left\|\nabla u_{0}\right\|_{L_{x}^{2}}+\mathcal{K}_{T}\|\nabla u\|_{L_{T}^{\infty} L_{x}^{2}}^{2}\|v\|_{L_{T}^{\infty} L_{x}^{2}},
\end{gathered}
$$

for all $0 \leq T \leq T_{\max }$.

Hence, by Gronwall inequality, we obtain

$$
\|\nabla v(t)\|_{L_{x}^{2}}^{2} \leq 2 \Phi^{2} e^{2 G(T)^{2} t}, \quad t \in\left[0, T_{\max }\right) .
$$

The estimate (3.14) proves Theorem 1.3.

We also have the following.

REMARK 3.1. Let $\mu>0$ and let $u_{\mu}(t), v_{\mu}(t)$ be the solutions of the initial value problem (1.1), with $u_{\mu} \in C\left(\left[0, T_{\max }\right], L_{x}^{2}\right)$. If the initial data $v_{0} \in L^{1}$, then we get

$$
\limsup _{\mu \rightarrow \infty}\left\|v_{\mu}(t)\right\|_{L^{1}} \leq\left\|u_{0}\right\|_{L^{2}}, \quad t \in\left[0, T_{\max }\right],
$$

and

$$
\left|\int_{\mathbb{R}^{n}} v_{\mu}(t, x) d x-\lambda\right|\left|u_{0} \|_{L^{2}}^{2}\right| \stackrel{\mu \rightarrow \infty}{\longrightarrow 0} .
$$


Proof. Observe that

$$
\left|v_{\mu}(t)\right| \leq e^{-\frac{t}{\mu}}\left|v_{0}\right|+\frac{|\lambda|}{\mu} \int_{0}^{t} e^{-\frac{t-s}{\mu}}\left|u_{\mu}(s)\right|^{2} d s .
$$

Integrating (3.15) and using the conserved quantity (1.2), we have

$$
\int_{\mathbb{R}^{n}}\left|v_{\mu}(x, t)\right| d x \leq e^{-\frac{t}{\mu}}\left(\left\|v_{0}\right\|_{1}-|\lambda||| u_{0} \|_{L^{2}}^{2}\right)+|\lambda|\left\|u_{0}\right\|_{L^{2}}^{2}, \quad \forall t \in\left[0, T_{\max }\right] .
$$

Similarly, we obtain

$$
\int_{\mathbb{R}^{n}} v(x, t) d x=e^{-\frac{t}{\mu}}\left(\int_{\mathbb{R}^{n}} v_{0}(x) d x-\lambda\left\|u_{0}\right\|_{L^{2}}^{2}\right)+\lambda\left\|u_{0}\right\|_{L^{2}}^{2}, \quad \forall t \in\left[0, T_{\max }\right] .
$$

Note that this equality proves the remark.

Lemma 3.1. Let $T>0,1 \leq p<q \leq \infty$ and $A, B \geq 0$. It follows that there exists $\Gamma=\Gamma(B, p, q, T)$ such that if $f \in L_{(0, T)}^{q}$ satisfies

$$
\|f\|_{L_{(0, t)}^{q}} \leq A+B\|f\|_{L_{(0, t)}^{p}},
$$

for all $0<t<T$, then

$$
\|f\|_{L_{(0, t)}^{q}} \leq A \Gamma
$$

Proof. See $[6]$.

REMARK 3.2. In order to estimate $\|u\|_{L_{x}^{4} L_{(0, t)}^{4}}$, we also could have used the Lemma 3.1.

In fact, let $0 \leq t \leq T \leq T_{\max }$ and let $v_{0} \in L^{2}, u_{0} \in L^{2}$. Since $(4,4)$ is an admissible pair in $\mathbb{R}^{2}$, using the integral equation for $u$ and the global well-posedness result in $L^{2} \times L^{2}$, we have

$$
\begin{aligned}
\|u\|_{L_{x}^{4} L_{(0, t)}^{4}} & \leq C\left\|u_{0}\right\|_{L^{2}}+\left\|\int_{0}^{t} S\left(t-t^{\prime}\right) u\left(t^{\prime}\right) v\left(t^{\prime}\right) d t^{\prime}\right\|_{L_{x}^{4} L_{(0, t)}^{4}} \\
& \leq C\left\|u_{0}\right\|_{L^{2}}+C\|u v\|_{L_{x}^{4 / 3} L_{(0, t)}^{4 / 3}} \\
& \leq C\left\|u_{0}\right\|_{L^{2}}+C\|u\|_{L_{(0, t)}^{4 / 3} L_{x}^{4}}\|v\|_{L_{(0, T)}^{\infty} L_{x}^{2} .}
\end{aligned}
$$

Now by Lemma 3.1 we conclude that

$$
\|u\|_{L_{x}^{4} L_{(0, t)}^{4}} \leq\left\|u_{0}\right\|_{L^{2}} \Gamma\left(\|v\|_{L_{(0, T)}^{\infty} L_{x}^{2}}, T\right) .
$$

4. Global well-posedness in $H^{s}\left(\mathbb{R}^{2}\right) \times L^{2}\left(\mathbb{R}^{2}\right), 2 / 3<s \leq 1$

In this section we will prove Theorem 1.4.

4.1. A priori Estimates. If one takes $\lambda=-1$ in (1.3), then the energy of the system is decreasing, i.e,

$$
E(u, v)=E(u(t), v(t)) \leq E\left(u_{0}, v_{0}\right), \quad \forall t \geq 0,
$$

where

$$
E(u, v)=\int_{\mathbb{R}^{n}}\left(|\nabla u|^{2}+2 v|u|^{2}+v^{2}\right) d x=\int_{\mathbb{R}^{n}}\left(|\nabla u|^{2}-|u|^{4}+\mu^{2}\left|v_{t}\right|^{2}\right) d x .
$$

From (1.2), (4.2) and the immersion 


$$
\|u(t)\|_{L_{x}^{4}} \leq c_{0}\|u(t)\|_{L_{x}^{2}}^{1 / 2}\|\nabla u(t)\|_{L_{x}^{2}}^{1 / 2}
$$

we get

$$
\begin{aligned}
\int_{\mathbb{R}^{2}}|\nabla u|^{2} d x+\int_{\mathbb{R}^{2}}|v|^{2} d x & \leq E\left(u_{0}, v_{0}\right)-2 \int_{\mathbb{R}^{2}}|u|^{2} v d x \\
& \leq E\left(u_{0}, v_{0}\right)+2\|v\|_{L_{x}^{2}}\|u\|_{L_{x}^{4}}^{2} \\
& \leq E\left(u_{0}, v_{0}\right)+2 c_{0}^{2}\|v\|_{L_{x}^{2}}\left\|u_{0}\right\|_{L_{x}^{2}}\|\nabla u(t)\|_{L_{x}^{2}} .
\end{aligned}
$$

Thus

$$
\frac{1}{2} \int_{\mathbb{R}^{2}}|\nabla u|^{2} d x+\left(1-2 c_{0}^{4}|| u_{0} \|_{L_{x}^{2}}^{2}\right) \int_{\mathbb{R}^{2}}|v|^{2} d x \leq E\left(u_{0}, v_{0}\right),
$$

which gives

$$
\int_{\mathbb{R}^{2}}|v|^{2} d x \leq 2 E\left(u_{0}, v_{0}\right), \quad \int_{\mathbb{R}^{2}}|\nabla u|^{2} d x \leq 2 E\left(u_{0}, v_{0}\right), \quad \forall t \geq 0
$$

since

$$
2 c_{0}^{2}\left\|u_{0}\right\|_{L_{x}^{2}} \leq 1
$$

In a similar way, by (4.2), the immersion (4.3) and (4.5), we get

$$
\begin{aligned}
\int_{\mathbb{R}^{2}} \mu^{2}\left|v_{t}\right|^{2} d x & \leq E\left(u_{0}, v_{0}\right)+\int_{\mathbb{R}^{2}}|u|^{4} d x \\
& \leq E\left(u_{0}, v_{0}\right)+c_{0}^{4}\left\|u_{0}\right\|_{L_{x}^{2}}^{2}\|\nabla u(t)\|_{L_{x}^{2}}^{2} \\
& \leq \frac{3}{2} E\left(u_{0}, v_{0}\right), \quad \text { if } \quad 2 c_{0}^{2}\left\|u_{0}\right\|_{L_{x}^{2}} \leq 1 .
\end{aligned}
$$

Moreover, by (4.3), (4.4) and (4.6), also is not dificult to see that

$$
\int_{\mathbb{R}^{2}}|u|^{4} d x \leq \frac{1}{2} E\left(u_{0}, v_{0}\right), \quad \text { if } \quad 2 c_{0}^{2}\left\|u_{0}\right\|_{L_{x}^{2}} \leq 1 .
$$

REMARK 4.1. 1) As a consequence of the immersion (4.3) it follows that if $c_{0}^{4}\left\|u_{0}\right\|_{L_{x}^{2}}^{2} \leq 1$, then

$$
E(u, v) \geq 0
$$

2) If $4 c_{0}^{2}\left\|u_{0}\right\|_{L_{x}^{2}} \leq 1$, then

$$
\int_{\mathbb{R}^{2}}|\nabla u|^{2} d x+\int_{\mathbb{R}^{2}}|v|^{2} d x \leq \frac{5}{3} \int_{\mathbb{R}^{2}}\left|\nabla u_{0}\right|^{2} d x+\frac{5}{3} \int_{\mathbb{R}^{2}}\left|v_{0}\right|^{2} d x .
$$

In fact, by (4.2) and (4.1), we have

$$
\begin{aligned}
\int_{\mathbb{R}^{2}}|\nabla u|^{2} d x & +\int_{\mathbb{R}^{2}}|v|^{2} d x \leq \int_{\mathbb{R}^{2}}\left|\nabla u_{0}\right|^{2} d x+\int_{\mathbb{R}^{2}}\left|v_{0}\right|^{2} d x \\
& +2 c_{0}^{2}\|v\|_{L_{x}^{2}}\left\|u_{0}\right\|_{L_{x}^{2}}\|\nabla u(t)\|_{L_{x}^{2}}+2 c_{0}^{2}\left\|v_{0}\right\|_{L_{x}^{2}}\left\|u_{0}\right\|_{L_{x}^{2}}|| \nabla u_{0} \|_{L_{x}^{2}},
\end{aligned}
$$

and using the Young inequality we deduce (4.9).

3) The integral representation (1.6) of $v$ and the Cauchy-Schwartz inequality give

$$
\|v(t)\|_{L_{x}^{2}} \leq e^{-t / \mu}\left\|v_{0}\right\|_{L^{2}}+\sqrt{\frac{1}{2 \mu}}\left(1-e^{-2 t / \mu}\right)^{1 / 2}\|u\|_{L_{[0, t]}^{4} L_{x}^{4}}^{2}, \quad t \geq 0
$$


GLOBAL WELL-POSEDNESS FOR THE CRITICAL SCHRÖDINGER-DEBYE SYSTEM 261

and the estimate (4.8) shows that if $2 c_{0}^{2}\left\|u_{0}\right\|_{L^{2}} \leq 1$, then

$$
\|v(t)\|_{L_{x}^{2}} \leq e^{-t / \mu}\left\|v_{0}\right\|_{L^{2}}+\sqrt{\frac{1}{\mu}} E\left(u_{0}, v_{0}\right)^{1 / 2}\left(1-e^{-2 t / \mu}\right)^{1 / 2} t^{1 / 2}, \quad t \geq 0 .
$$

4.2. Iteration. Now let $v_{0} \in L^{2}$ and $u_{0} \in H^{s}, 2 / 3<s<1$, be the initial data of the IVP (1.1), with the small condition (4.6), i.e.,

$$
2 c_{0}^{2}\left\|u_{0}\right\|_{L_{x}^{2}}<1
$$

Fix a large time $T$ and let $N=N(T)$ be a cutoff (to be specified). Write (4.13)

$$
u_{0}:=\omega_{0}+\eta_{0} \quad \text { with } \quad \omega_{0}=\int_{|\xi|<N} e^{i x \cdot \xi} \widehat{u_{0}}(\xi) d \xi, \quad \eta_{0}=\int_{|\xi| \geq N} e^{i x \cdot \xi} \widehat{u_{0}}(\xi) d \xi .
$$

We observe that

$$
\left\|\omega_{0}\right\|_{\dot{H}^{\theta}}=\left\{\int_{|\xi|<N}|\xi|^{2 \theta}\left|\widehat{u_{0}}(\xi)\right|^{2} d \xi\right\}^{1 / 2}<\left\|u_{0}\right\|_{\dot{H}^{s}} N^{\theta-s}, \quad \text { for any } \theta \geq s .
$$

Similarly, we get

$$
\left\|\eta_{0}\right\|_{\dot{H}^{\sigma}}=\left\{\int_{|\xi| \geq N}|\xi|^{2 \sigma}\left|\widehat{u_{0}}(\xi)\right|^{2} d \xi\right\}^{1 / 2} \leq\left\|u_{0}\right\|_{\dot{H}^{s}} N^{\sigma-s}, \quad \text { if } \sigma \leq s,
$$

and

$$
\left\|\omega_{0}\right\|_{L_{x}^{2}} \leq\left\|u_{0}\right\|_{L_{x}^{2}}
$$

In particular, these estimates proves that $w_{0} \in H^{\infty}$ and $\eta_{0} \in H^{s}$. Now we consider the IVP

$$
\left\{\begin{array}{l}
i \stackrel{0}{u}_{t}+\frac{1}{2} \Delta \stackrel{0}{u}=\stackrel{00}{u} v \\
\mu \stackrel{0}{v}_{t}+\stackrel{0}{v}=-|\stackrel{0}{u}|^{2} \\
\stackrel{0}{u}(0)=\omega_{0} \text { and } \stackrel{0}{v}(0)=v_{0}
\end{array}\right.
$$

where $w_{0}$ is defined in (4.13) and verifies (4.14) and (4.16). We know by the Theorem 1.2 that if the initial data $\left(u_{0}, v_{0}\right) \in H^{1}\left(\mathbb{R}^{2}\right) \times L^{2}\left(\mathbb{R}^{2}\right)$, then there exists a unique solution $(u, v) \in C\left([0, T] ; H^{1}\left(\mathbb{R}^{2}\right) \times L^{2}\left(\mathbb{R}^{2}\right)\right)$ of IVP (1.1). Shortly problem (4.17) is globally well-posedness in $H^{1}\left(\mathbb{R}^{2}\right) \times L^{2}\left(\mathbb{R}^{2}\right)$.

We write the solution $(u, v)$ of the system (1.1), as

$$
u=\stackrel{0}{u}+\stackrel{0}{\eta} \quad \text { and } \quad v=\stackrel{0}{v}+\stackrel{0}{z}
$$

where $(\stackrel{0}{\eta}, \stackrel{0}{z})$ satisfies the IVP

$$
\left\{\begin{array}{l}
\stackrel{i}{i \eta_{t}}+\frac{1}{2} \Delta \stackrel{0}{\eta}=(\stackrel{0}{u}+\stackrel{0}{\eta})(\stackrel{0}{v}+\stackrel{0}{z})-\stackrel{0}{u} v \\
\mu \stackrel{0}{z_{t}}+\stackrel{0}{z}=-|\stackrel{0}{u}+\stackrel{0}{\eta}|^{2}+|\stackrel{0}{u}|^{2} \\
\stackrel{0}{\eta}(0)=\eta_{0}, \quad \stackrel{0}{z}(0)=z_{0}=0
\end{array}\right.
$$


where $\eta_{0}$ is defined in (4.13) and verifies (4.15).

Consider the IVP (4.19) in the following integral form

$$
\stackrel{0}{\eta}(t)=e^{i t \Delta / 2} \eta_{0}+\stackrel{0}{\psi}(t),
$$

where

$$
\stackrel{0}{\psi}(t)=i \int_{0}^{t} e^{i \Delta(t-\tau) / 2}(\stackrel{0}{u} \underset{z}{z}+\stackrel{0}{\eta} \stackrel{0}{v}+\stackrel{0}{\eta} \underset{z}{z}) d \tau
$$

and

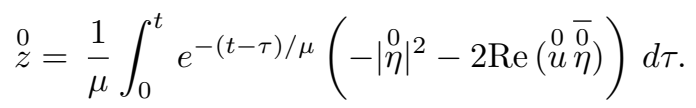

We have the following LWP result

THEOREM 4.1. Let $\left(\tilde{\omega}_{0}, \tilde{v}_{0}\right) \in H^{s_{1}}\left(\mathbb{R}^{2}\right) \times H^{s_{2}}\left(\mathbb{R}^{2}\right)$, where $\left(s_{1}, s_{2}\right)$ satisfy the condition (1.7). Then, for all $T$ such that

$$
0<T \leq \frac{c}{\left\|\tilde{\omega}_{0}\right\|_{H^{s_{1}}}^{2}+\left\|\tilde{v}_{0}\right\|_{H^{s_{2}}}^{2}},
$$

there exists a unique solution

$$
(\stackrel{0}{u}, \stackrel{0}{v}) \in C\left([0, T] ; H^{s_{1}}\left(\mathbb{R}^{2}\right) \times H^{s_{2}}\left(\mathbb{R}^{2}\right)\right)
$$

to the initial value problem (4.17) with initial data $\left(\tilde{\omega}_{0}, \tilde{v}_{0}\right)$ such that:

$$
\left\|\vartheta_{T}(t) \stackrel{0}{u}(\cdot, t)\right\|_{X_{s_{1}, b_{1}}}+\left\|\vartheta_{T}(t) \stackrel{0}{v}(\cdot, t)\right\|_{H_{s_{2}, b_{2}}} \leq c\left\|\tilde{\omega}_{0}\right\|_{H^{s_{1}}}+c\left\|\tilde{v}_{0}\right\|_{H^{s_{2}}},
$$

wehere $\vartheta_{T}$ is defined in (2.1) and for some suitable $b_{1}>1 / 2$ and $b_{2}>1 / 2$.

Proof. It follows immediately from the proof of Theorem 1.1 in Corcho, Oliveira and Silva $[\mathbf{9}]$.

We also have the following.

Theorem 4.2. Let $u_{0}$ be in $H^{s}\left(\mathbb{R}^{2}\right)$ and let $\eta_{0}$ be as defined in (4.13). Then, there exists $t_{1}>0$ such that

$$
t_{1}=N^{-2(1-s)-},
$$

and there exists a unique solution

$$
(\stackrel{0}{\eta}, \stackrel{0}{z}) \in C\left(\left[0, t_{1}\right] ; H^{s}\left(\mathbb{R}^{2}\right) \times L^{2}\left(\mathbb{R}^{2}\right)\right)
$$

to the initial value problem (4.19) with initial data $\eta_{0}$ and $z_{0}=0$, such that

$$
\left.\left\|\vartheta_{t_{1}}(t){ }^{0} \eta(\cdot, t)\right\|\right|_{X_{s, b}} \leq c\left\|\eta_{0}\right\|_{H^{s}},
$$

for some suitable $b>1 / 2$.

Proof. The proof is very similar to the proof of Theorem 1.1 and to the proof of Theorem 4.1 in Corcho, Oliveira and Silva [9].

REMARK 4.2. If in Theorem 4.1 we take $s_{1}=1, s_{2}=0$ and if also we consider $\tilde{\omega}_{0}=\omega_{0}$ as defined in (4.13) and $\tilde{v}_{0}=v_{0}$, then we can take $T=t_{1}=N^{-2(1-s)-}$ and thus obtain the same existence interval $\left[0, t_{1}\right]$, for the systems (4.17) and (4.19). 
Note that by $(4.14)$, we have $\left\|\nabla \omega_{0}\right\|_{L_{x}^{2}\left(\mathbb{R}^{2}\right)} \lesssim\left\|\omega_{0}\right\|_{\dot{H}^{1}\left(\mathbb{R}^{2}\right)} \leq\left\|u_{0}\right\|_{\dot{H}^{s}} N^{1-s}$. From (4.16) it follows that

$$
\begin{aligned}
E\left(\omega_{0}, v_{0}\right)= & \int_{\mathbb{R}^{2}}\left(\left|\nabla \omega_{0}\right|^{2}+2\left|\omega_{0}\right|^{2} v_{0}+\left|v_{0}\right|^{2}\right) d x \leq\left\|\nabla \omega_{0}\right\|_{L_{x}^{2}\left(\mathbb{R}^{2}\right)}^{2}+\left\|v_{0}\right\|_{L_{x}^{2}\left(\mathbb{R}^{2}\right)}^{2} \\
& +c_{0}|| \omega_{0}||_{L_{x}^{2}\left(\mathbb{R}^{2}\right)}|| \nabla \omega_{0}\left\|_{L_{x}^{2}\left(\mathbb{R}^{2}\right)}|| v_{0}\right\|_{L_{x}^{2}\left(\mathbb{R}^{2}\right)} \\
\lesssim_{s} & N^{2(1-s)} .
\end{aligned}
$$

Integrating the inequality (4.8), considering the time $t_{1}$ as in (4.25), we have

$$
\int_{0}^{t_{1}} \int_{\mathbb{R}^{2}}|u|^{4} d x d t \leq \frac{1}{2} E\left(u_{0}, v_{0}\right) t_{1} \leq 1
$$

By Remark (4.2) in the time interval $\left[0, t_{1}\right]$ we have local existence for both systems (4.17) and (4.19).

Now from (4.16), $2 c_{0}^{2}\left\|w_{0}\right\|_{L_{x}^{2}} \leq 2 c_{0}^{2}\left\|u_{0}\right\|_{L_{x}^{2}}<1$, thus by (4.5) we have

$$
\left\|\nabla u^{0}\left(t_{1}\right)\right\|_{L_{x}^{2}\left(\mathbb{R}^{2}\right)} \lesssim s N^{(1-s)}
$$

and

$$
\left\|{ }^{0}\left(t_{1}\right)\right\|_{L_{x}^{2}\left(\mathbb{R}^{2}\right)}{ }_{s} N^{(1-s)}
$$

Furthemore, the immersion (4.3), conservation law (1.2), inequalities (4.29) above and (4.36) below imply that

$$
\left\|{ }^{0}\left(t_{1}\right)\right\|_{L_{x}^{4}} \lesssim N^{(-3 s / 4)+} \quad \text { and } \quad\left\|u\left(t_{1}\right)\right\|_{L_{x}^{4}} \lesssim_{s} N^{(1-s) / 2} .
$$

For $t \in\left[0, t_{1}\right]$, by $(4.18)$ and $(4.20)$, we have

$$
u(t)=\stackrel{0}{u}(t)+\stackrel{0}{\psi}(t)+e^{i t \Delta / 2} \eta_{0} \quad \text { and } \quad v(t)={ }_{0}^{0}(t)+\stackrel{0}{z}(t),
$$

where $(\stackrel{0}{u}, \stackrel{0}{v})$ is the solution of $(4.17)$ and $(\stackrel{0}{\eta}, \stackrel{0}{z})=\left(\psi+e^{i t \Delta / 2} \eta_{0}, \stackrel{0}{z}\right)$ is the solution of (4.19). Now we define the new intial data for the second iteration

$$
\begin{aligned}
& \omega_{1}=\stackrel{0}{u}\left(t_{1}\right)+\stackrel{0}{\psi}\left(t_{1}\right) \quad \text { and } \quad v_{1}={ }_{v}^{0}\left(t_{1}\right)+{ }^{0}\left(t_{1}\right), \\
& \eta_{1}=e^{i t_{1} \Delta / 2} \eta_{0} \quad \text { and } \quad z_{1}=0 .
\end{aligned}
$$

In each iteration we consider the decomposition of the initial data as in (4.33). Therefore $\eta_{1}, \ldots, \eta_{k}=e^{i k t \Delta / 2} \eta_{0}$ have the same properties of $\eta_{0}$ with $\left\|\eta_{k}\right\|_{H^{s}}=$ $\left\|\eta_{0}\right\|_{H^{s}}$ and $z_{1}=\cdots=z_{k}=0$. We hope that $\omega_{1}, \ldots, \omega_{k}$ and $v_{1}, \ldots, v_{k}$ also have the same properties of $\omega_{0}$ and $v_{0}$ respectively in order to ensure the same existence interval $\left[0, t_{1}\right]$ in each iteration and attach the existence interval $[0, T]$, extending the solution of the systems (4.17) and (4.19). This fact is proved by induction. Here we will prove only the case $k=1$ and note that a similar argument works in the general case.

From (4.1), we have

$$
E\left(\stackrel{0}{u}\left(t_{1}\right), \stackrel{0}{v}\left(t_{1}\right)\right) \leq E(\stackrel{0}{u}(0), \stackrel{0}{v}(0))=E\left(\omega_{0}, v_{0}\right) .
$$


Thus we get

$$
E\left(\omega_{1}, v_{1}\right) \leq E\left(\omega_{0}, v_{0}\right)+\left[E\left(\omega_{1}, v_{1}\right)-E\left(\stackrel{0}{u}\left(t_{1}\right), \stackrel{0}{v}\left(t_{1}\right)\right)\right] .
$$

Using the immersion (4.3) and (4.33), we obtain

$$
\begin{aligned}
& \left|E\left(\omega_{1}, v_{1}\right)-E\left(\stackrel{0}{u}\left(t_{1}\right), \stackrel{0}{v}\left(t_{1}\right)\right)\right|=\left|E\left(\stackrel{0}{u}\left(t_{1}\right)+\stackrel{0}{\psi}\left(t_{1}\right), \stackrel{0}{v}\left(t_{1}\right)+\stackrel{0}{z}\left(t_{1}\right)\right)-E\left(\stackrel{0}{u}\left(t_{1}\right), \stackrel{0}{v}\left(t_{1}\right)\right)\right| \\
& \leq\left\|\nabla{ }^{0}\left(t_{1}\right)\right\|_{L_{x}^{2}}^{2}+2\left\|\nabla^{0} \psi\left(t_{1}\right)\right\|_{L_{x}^{2}}\left\|\nabla^{0} u\left(t_{1}\right)\right\|_{L_{x}^{2}}+2\|\|^{0}\left(t_{1}\right)\left\|_{L_{x}^{2}\left(\mathbb{R}^{2}\right)}\right\| \psi^{0} \psi\left(t_{1}\right) \|_{L_{x}^{4}}^{2} \\
& +4\left\|u^{0}\left(t_{1}\right)\right\|_{L_{x}^{4}}\left\|\psi^{0}\left(t_{1}\right)\right\|_{L_{x}^{4}}\left(\left\|v^{0}\left(t_{1}\right)\right\|_{L_{x}^{2}}+\left\|z^{0}\left(t_{1}\right)\right\|_{L_{x}^{2}}\right)+2\|\|^{0}\left(t_{1}\right)\left\|_{L_{x}^{2}}\right\| \psi\left(t_{1}\right) \|_{L_{x}^{4}}^{2}
\end{aligned}
$$

$$
+\left\|z^{0}\left(t_{1}\right)\right\|_{L_{x}^{2}}^{2}+2\left\|v^{0}\left(t_{1}\right)\right\|_{L_{x}^{2}}\left\|z^{0}\left(t_{1}\right)\right\|_{L_{x}^{2}}+2\left\|\left.\right|_{z} ^{0}\left(t_{1}\right)\right\|_{L_{x}^{2}}\left\|u\left(t_{1}\right)\right\|_{L_{x}^{4}} .
$$

In order to estimate (4.35), initially we will assume the following result, which be will proved later.

LEMMA 4.1. Let $\stackrel{0}{\eta}(t)$ be a solution of the IVP (4.19), and let $\stackrel{0}{\psi}(t)$ be the forcing term as defined in (4.20) and (4.21), then we have the following estimates

$$
\left\|{ }^{0} \psi(t)\right\|_{L_{x}^{2}\left(\mathbb{R}^{2}\right)} \leq c N^{-s} \quad \text { and } \quad\|\nabla \psi(t)\|_{L_{x}^{2}\left(\mathbb{R}^{2}\right)} \leq c N^{(-s / 2)^{+}},
$$

and also that

$$
\|\eta\|_{L_{t}^{4} L_{x}^{4}\left(\left[0, t_{1}\right] \times \mathbb{R}^{2}\right)} \leq c N^{-s}
$$

Using (4.22), the Minkowsky and the Cauchy-Schwartz inequalities, together with (4.28) and (4.37), for any $t \in\left[0, t_{1}\right]$ we have

$$
\begin{aligned}
\|z(t)\|_{L_{x}^{2}\left(\mathbb{R}^{2}\right)} & \leq \frac{1}{\mu} \int_{0}^{t_{1}} e^{-(t-\tau) / \mu}\left(\|\eta(\tau)\|_{L_{x}^{4}\left(\mathbb{R}^{2}\right)}^{2}+2\|\eta(\tau)\|_{L_{x}^{4}\left(\mathbb{R}^{2}\right)}\|u(\tau)\|_{L_{x}^{4}\left(\mathbb{R}^{2}\right)}\right) \\
& \lesssim \mu \mu \eta \eta\left\|_{L_{\left[0, t_{1}\right]}^{4} L_{x}^{4}}^{2}+\right\| \eta\left\|_{L_{\left[0, t_{1}\right]}^{4} L_{x}^{4}}\right\| u \|_{L_{\left[0, t_{1}\right]}^{4} L_{x}^{4}}^{0} \\
& \lesssim_{\mu}^{0}\|\eta\|_{L_{\left[0, t_{1}\right]}^{4} L_{x}^{4}}^{2}+\|\eta\|_{L_{\left[0, t_{1}\right]}^{4} L_{x}^{4}} \\
& \lesssim_{\mu} N^{-s} .
\end{aligned}
$$

From (4.29), (4.30), (4.31), (4.35), (4.36) and (4.39) we obtain

$$
\begin{aligned}
& \left|E\left(\omega_{1}, v_{1}\right)-E\left(\stackrel{0}{0}\left(t_{1}\right), \stackrel{0}{v}\left(t_{1}\right)\right)\right| \lesssim N^{(-s)^{+}}+N^{1-s} N^{(-s / 2)^{+}} \\
& +N^{(1-s)} N^{(-3 s / 2)^{+}}+N^{(1-s) / 2} N^{(-3 s / 4)^{+}}\left(N^{(1-s)}+N^{-s}\right) \\
& +N^{-s} N^{(-3 s / 2)^{+}}+N^{-2 s}+N^{1-s} N^{-s}+N^{1-s} N^{-s} \lesssim N^{((2-3 s) / 2)^{+}} .
\end{aligned}
$$

Combining (4.27), (4.34) and (4.40), we get that

$$
E\left(\omega_{1}, v_{1}\right) \leq E\left(\omega_{0}, v_{0}\right)+c N^{((2-3 s) / 2)^{+}} .
$$

Also, observe that by conservation quantity (1.2) and Lemma 4.1, we have

$$
\left\|\omega_{1}\right\|_{L^{2}} \leq\left\|u\left(t_{1}\right)\right\|_{L^{2}}+\left\|\psi\left(t_{1}\right)\right\|_{L^{2}} \leq\left\|\omega_{0}\right\|_{L^{2}}+c N^{-s} \leq\left\|u_{0}\right\|_{L^{2}}+c N^{-s} .
$$


Thus, the small condition (4.12) remains valid in the second iteration if

$$
2 c_{0}^{2}\left\|\omega_{1}\right\|_{L^{2}} \leq 2 c_{0}^{2}\left(\left\|u_{0}\right\|_{L^{2}}+c N^{-s}\right)<1,
$$

i.e., if $2 c_{0}^{2} c N^{-s}<1-2 c_{0}^{2}\left\|u_{0}\right\|_{L^{2}}$, which happens indeed if $N$ is very large. Also from (4.5), it follows that

$$
\left\|v_{1}\right\|_{L^{2}} \leq\left\|v\left(t_{1}\right)\right\|_{L^{2}}+\left\|z\left(t_{1}\right)\right\|_{L^{2}} \leq \sqrt{2 E\left(\omega_{0}, v_{0}\right)}+c N^{-s} \leq c N^{1-s} .
$$

The number of steps in the iteration is

$$
\frac{T}{t_{1}} \sim T N^{2(1-s)^{+}}
$$

Thus, by (4.27), we need that

$$
T N^{2(1-s)^{+}} N^{((2-3 s) / 2)^{+}}<E\left(\omega_{0}, v_{0}\right) \sim N^{2(1-s)},
$$

which is posible if $s>2 / 3$ and

$$
N=N(T)=T^{2^{+} /(3 s-2)}, \quad \text { or equivalently } \quad T=N^{(3 s-2) /\left(2^{+}\right)} .
$$

Observe also that the small condition remains valid in each iteration since, in similar way as in (4.43), we have

$$
\begin{aligned}
T N^{2(1-s)^{+}} 2 c_{0}^{2} c N^{-s} & =N^{(3 s-2) /\left(2^{+}\right)} N^{2(1-s)^{+}} 2 c_{0}^{2} c N^{-s}=2 c_{0}^{2} c N^{(2-3 s) / 2} \\
& <1-2 c_{0}^{2}\left\|u_{0}\right\|_{L^{2}}
\end{aligned}
$$

and similarly as in (4.44)

$$
\sqrt{2 E\left(\omega_{0}, v_{0}\right)}+T N^{2(1-s)^{+}} c N^{-s} \leq \sqrt{2 E\left(\omega_{0}, v_{0}\right)}+N^{(3 s-2) / 2^{+}} N^{2(1-s)^{+}} c N^{-s}
$$

$$
\leq c N^{1-s}
$$

and the inequalities (4.45), (4.46) are true if $N$ is very large and $s>2 / 3$.

4.3. Proof of Lemma 4.1. First we will prove the inequality (4.37). Since $(4,4)$ is an admissible pair of the group $\left\{e^{i t \Delta / 2}\right\}$, by $(4.20)$ and Prosition 2.4, it follows that

$$
\|\eta\|_{L_{\left[0, t_{1}\right]}^{4} L_{x}^{4}} \leq c\left\|\eta_{0}\right\|_{L_{x}^{2}}+\|\stackrel{0}{\psi}\|_{L_{t}^{4} L_{x}^{4}\left(\left[0, t_{1}\right] \times \mathbb{R}^{2}\right)} .
$$

Moreover, Proposition 2.4, the equality (4.21) and the Hölder inequality show that

$$
\begin{aligned}
\|\stackrel{0}{\psi}\|_{L_{t}^{4} L_{x}^{4}\left(\left[0, t_{1}\right] \times \mathbb{R}^{2}\right)} & \lesssim\left\|u^{0} \stackrel{0}{z}+\stackrel{0}{\eta} \stackrel{0}{v}+\stackrel{0}{\eta} \underset{z}{z}\right\|_{L_{\left[0, t_{1}\right]}^{4 / 3} L_{x}^{4 / 3}} \\
& \lesssim\|u\|_{L_{\left[0, t_{1}\right]}^{4} L_{x}^{4}}\|\|^{0}\left\|_{L_{\left[0, t_{1}\right]}^{2} L_{x}^{2}}+\right\| \eta\left\|_{L_{\left[0, t_{1}\right]}^{4}} L_{x}^{4}\right\|\left\|^{0}\right\|_{L_{\left[0, t_{1}\right]}^{2} L_{x}^{2}} \\
& +\|\eta\|_{L_{\left[0, t_{1}\right]}^{4} L_{x}^{4}}\|z\|_{L_{\left[0, t_{1}\right]}^{2} L_{x}^{2} .}
\end{aligned}
$$

By estimates (4.5) and (4.27), we get

$$
\|v(t)\|_{L_{x}^{2}} \leq \sqrt{2 E\left(\omega_{0}, v_{0}\right)} \lesssim N^{1-s}, \quad t \geq 0
$$


Therefore, combining (4.28), (4.38), (4.48) and (4.49), we obtain

$$
\begin{aligned}
& \|\stackrel{0}{\|}\|_{L_{\left[0, t_{1}\right]}^{4} L_{x}^{4}} \lesssim t_{1}^{1 / 2}\|z\|_{L_{\left[0, t_{1}\right]}^{\infty} L_{x}^{2}}+t_{1}^{1 / 2}\|\eta\|_{L_{\left[0, t_{1}\right]}^{4}} L_{x}^{4}\|v\|_{L_{\left[0, t_{1}\right]}^{\infty} L_{x}^{2}}^{0} \\
& \quad+t_{1}^{1 / 2}\|\eta\|_{L_{\left[0, t_{1}\right]}^{4} L_{x}^{4}}^{0}\|z\|_{L_{\left[0, t_{1}\right]}^{\infty} L_{x}^{2}} \\
& \lesssim t_{1}^{1 / 2}\|\eta\|_{L_{\left[0, t_{1}\right]}^{4} L_{x}^{4}}\left(N^{1-s}+\|\eta\|_{L_{\left[0, t_{1}\right]}^{4} L_{x}^{4}}+\|\eta\|_{L_{\left[0, t_{1}\right]}^{4} L_{x}^{4}}^{2}\right) .
\end{aligned}
$$

Note that $t_{1}^{1 / 2}=N^{-(1-s)^{-}}, 1 \ll N$. Thus, it follows from (4.47) and (4.50) that

$$
\|\eta\|_{L_{\left[0, t_{1}\right]}^{4} L_{x}^{4}} \leq c\left\|\eta_{0}\right\|_{L_{x}^{2}}+c t_{1}^{1 / 2}\|\eta\|_{L_{\left[0, t_{1}\right]}^{4} L_{x}^{4}}^{2}\left(1+\|\eta\|_{L_{\left[0, t_{1}\right]}^{4} L_{x}^{4}}\right),
$$

and from a standard continuity argument it follows that

$$
\|\eta\|_{L_{t}^{4} L_{x}^{4}\left(\left[0, t_{1}\right] \times \mathbb{R}^{2}\right)} \leq 2 c\left\|\eta_{0}\right\|_{L_{x}^{2}} \lesssim N^{-s} .
$$

Now we will prove the first inequality in $(4.36)$. Since $(\infty, 2)$ and $(4,4)$ are admissible pairs of the group $\left\{e^{i t \Delta / 2}\right\}$, using (4.21), (4.48)-(4.51) it follows that

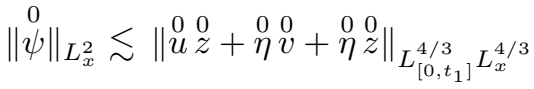

$$
\begin{aligned}
& \lesssim t_{1}^{1 / 2}\|\eta\|_{L_{\left[0, t_{1}\right]}^{4} L_{x}^{4}}\left(N^{1-s}+\|\eta\|_{L_{\left[0, t_{1}\right]}^{4} L_{x}^{4}}+\|\eta\|_{L_{\left[0, t_{1}\right]}^{4} L_{x}^{4}}^{2}\right) \\
& \lesssim N^{-s} \text {. }
\end{aligned}
$$

Finally we will prove the second inequality in (4.36). By Theorem 2.1, it follows that

$$
\begin{aligned}
& \|\nabla \stackrel{0}{\psi}(t)\|_{L_{x}^{2}\left(\mathbb{R}^{2}\right)} \leq c\|\stackrel{0}{\psi}\|_{X_{1, b}} \\
& \leq c\|\stackrel{0}{u} \stackrel{0}{z}+\stackrel{0}{\eta} \stackrel{0}{v}+\stackrel{0}{\eta} \stackrel{0}{z}\|_{X_{1, b-1}} \\
& =c \sup _{\|W\|_{X_{\{-1,1-b\}} \leq 1}}\left|\int_{\mathbb{R}_{t} \times \mathbb{R}_{x}^{2}}\left(\begin{array}{l}
0 \\
u \\
z
\end{array}+\stackrel{0}{\eta} \stackrel{0}{v}+\stackrel{0}{\eta} \underset{z}{z}\right) \bar{W} d x d t\right|
\end{aligned}
$$

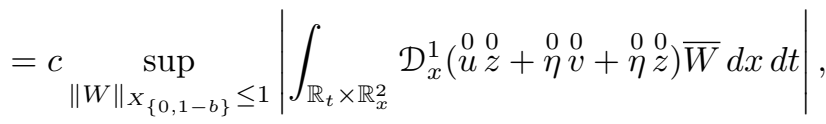

where $\widehat{\left(\mathcal{D}_{x}^{s} f\right)}(\xi)=\langle\xi\rangle^{s} \widehat{f}(\xi), \mathcal{D}_{x}^{1}:=\mathcal{D}_{x}$. Without loss of generality we only consider the term with ${ }_{0}^{0} \underset{z}{0}$, because the estimates on the other terms in (4.53) are similar or better. Using the Plancherel equality and the Hölder inequality, we have

$$
\begin{aligned}
& \left|\int_{\mathbb{R}_{t} \times \mathbb{R}_{x}^{2}} \mathcal{D}_{x}(\stackrel{0}{u} \stackrel{0}{z}) \bar{W} d x d t\right| \leq\left|\int_{\mathbb{R}_{t} \times \mathbb{R}_{x}^{2}} \mathcal{D}_{x}(\stackrel{0}{u}) \stackrel{0}{z} \bar{W} d x d t\right|+\mid \int_{\mathbb{R}_{t} \times \mathbb{R}_{x}^{2}} \stackrel{0}{u} \mathcal{D}_{x}\left(\begin{array}{l}
z \\
)
\end{array} \bar{W} d x d t \mid\right. \\
& \leq\|z\|_{L_{t_{1}}^{2+} L_{x}^{2+}}\left\|\mathcal{D}_{x}(\stackrel{0}{u})\right\|_{L_{t_{1}}^{4} L_{x}^{4}}\|W\|_{L_{t_{1}}^{4-} L_{x}^{4-}}+\left|\int_{\mathbb{R}_{t} \times \mathbb{R}_{x}^{2}} \mathcal{D}_{x}^{1 / 2^{+}}\left(\begin{array}{l}
z \\
z
\end{array}\right) \mathcal{D}_{x}^{1 / 2^{-}}(\bar{W} \stackrel{0}{u}) d x d t\right| \\
& \leq\|z\|_{L_{t_{1}}^{2+} L_{x}^{2+}}\left\|\mathcal{D}_{x}(\stackrel{0}{u})\right\|_{L_{t_{1}}^{4} L_{x}^{4}}\|W\|_{L_{t_{1}}^{4-} L_{x}^{4-}}+\left\|\mathcal{D}_{x}^{1 / 2^{+}}(\stackrel{0}{z})\right\|_{L_{t_{1}}^{2} L_{x}^{2}}\left\|\mathcal{D}_{x}^{1 / 2^{-}}(\bar{W} u)\right\|_{L_{t_{1}}^{2} L_{x}^{2}}
\end{aligned}
$$

$$
:=I_{1} I_{2} I_{3}+I_{4} I_{5} \text {. }
$$


Now we will estimate all the terms in (4.54):

(1) Estimate of $I_{5}$ :

Observe that the local well-posedness theory in Theorem 4.1, the conservation quantity (1.2) and a priori estimates (4.5) imply that

$$
\left\|\vartheta_{t_{1}}(t) \stackrel{0}{u}(\cdot, t)\right\|\left\|_{X_{1, b}} \leq c\right\| \omega_{0}\left\|_{H^{1}}+c\right\| v_{0} \|_{L^{2}} \leq c N^{1-s},
$$

and

$$
\left\|\vartheta_{t_{1}}(t) \stackrel{0}{u}(\cdot, t)\right\|_{X_{0, b}} \leq c\left\|\omega_{0}\right\|_{L^{2}}+c\left\|v_{0}\right\|_{L^{2}} \leq c .
$$

Using the Proposition 2.1 item (4), interpolation, (4.55) and (4.56), we have

$$
\begin{aligned}
I_{5} & =\left\|\mathcal{D}_{x}^{1 / 2^{-}}(\bar{W} u)\right\|_{L_{t_{1} L_{x}^{2}}^{2}} \leq c\|u\|_{X_{1 / 2,1 / 2+}}\|\bar{W}\|_{X_{0,1 / 2}} \\
& \leq c\|u\|_{X_{1,1 / 2+}}^{1 / 2}\|0\|_{X_{0,1 / 2}+}^{1 / 2} \\
& \leq c N^{(1-s) / 2} .
\end{aligned}
$$

(2) Estimate of $I_{3}$ :

Using the Proposition 2.2 item ii) (because $(4,4)$ is an admisible pair) we have

$$
\|W\|_{L_{x, t}^{4}} \leq c\|W(\cdot, t)\|_{X_{0,1 / 2}+} .
$$

Interpolating this inequality with

$$
\|W\|_{L_{x, t}^{2}} \leq c\|W(\cdot, t)\|_{X_{0,0}}
$$

we obtain

$$
I_{3}=\|W\|_{L_{t_{1}}^{4-} L_{x}^{4-}} \leq c\|W(\cdot, t)\|_{X_{0,1 / 2^{-}}} \leq c .
$$

(3) Estimate of $I_{2}$ :

To estimate $I_{2}$ we also will use the Proposition 2.2 item ii). Thus

$$
I_{2}=\left\|\mathcal{D}_{x}{ }^{0} u\right\|_{L_{t_{1}}^{4} L_{x}^{4}} \leq c\left\|\vartheta_{t_{1}}{ }^{0}\right\|_{X_{1,1 / 2}+} \leq c N^{1-s} .
$$

(4) Estimate of $I_{1}$ and $I_{4}$ :

By Theorem 4.2 and the bilinear estimates in Proposition 2.4 of Corcho, Oliveira and Silva $[\mathbf{9}]$ for $s>1 / 2^{+}$we have that

$$
\left\|\vartheta_{t_{1}}{ }^{2}\right\|_{H_{s, 1 / 2}} \leq c\left\|\eta_{0}\right\|_{H^{s}} \leq c\left\|u_{0}\right\|_{H^{s}} .
$$

From Gagliardo-Nirenberg inequality and (4.39), we get

$$
I_{1}=\|z\|_{L_{t_{1}}^{2+} L_{x}^{2+}}^{0}=t_{1}^{1 / 2^{+}}\|z\|_{L_{t_{1}}^{\infty} L_{x}^{2+}}^{0} \leq t_{1}^{1 / 2}\|z\|_{L_{t_{1}}^{\infty} L_{x}^{2}}^{1-\theta}\left\|\mathcal{D}_{x}^{s} z\right\|_{L_{t_{1}}^{\infty} L_{x}^{2}}^{\theta} \lesssim_{\mu} N^{-1^{+}},
$$

where $2 \theta=0^{+} /\left(2^{+}\right)$. Finally, using interpolating and (4.58), we obtain

$$
\begin{aligned}
I_{4}=\left\|\mathcal{D}_{x}^{1 / 2^{+}}(\underset{z}{z})\right\|_{L_{t_{1}}^{2} L_{x}^{2}} & \leq t_{1}^{1 / 2}\left\|\mathcal{D}_{x}^{s} z\right\|_{L_{t_{1}}^{\infty} L_{x}^{2}}^{\theta_{1}}\|z\|_{L_{t_{1}}^{\infty} L_{x}^{2}}^{1-\theta_{1}} \leq c t_{1}^{1 / 2}\|z\|_{L_{t_{1}}^{\infty} L_{x}^{2}}^{1-\theta_{1}} \\
& \lesssim_{\mu} N^{(-1 / 2)^{+}},
\end{aligned}
$$

where $s \theta_{1}=(1 / 2)^{+}$.

Combining (4.53), (4.54) and the estimates on $I_{1}, \ldots, I_{5}$, we have

$$
\|\nabla \stackrel{0}{\psi}(t)\|_{L_{x}^{2}\left(\mathbb{R}^{2}\right)} \leq c N^{(-s / 2)^{+}} .
$$


REMARK 4.3. 1) By (4.4), the condition (4.12) can be replaced by the weaker condition:

$$
\sqrt{2} c_{0}^{2}\left\|u_{0}\right\|_{L_{x}^{2}}<1 .
$$

2) The inequality (4.35) shows that a better estimate for $\|\nabla \psi(\cdot)\|_{L_{x}^{2}}$ implies a better GWP result.

Acknowledgements The authors are thankful to the anonymous referee for his or her valuable corrections and comments on early version of this work that helped to improve the presentation and also to Adan Corcho by bring the problem of this work for our attention. The first author was supported by FAPESP, Brazil under grant 2012/23054-6 and by the National Council of Technological and Scientific Development (CNPq), Brazil, by the grant 481715/2012-6.

\section{References}

[1] J. Berg and J. Löfström, Interpolation spaces, Springer, Berlin, (1976).

[2] J. Bourgain, Fourier restriction phenomena for certain lattice subsets and applications to nonlinear evolution equations. Parts I, II, Geometric and Funct. Anal., 3 (1993), 107-156, 209-262.

[3] J. Bourgain, Global solutions of nonlinear Schrödinger equations, American Mathematical Society, Colloquium Publications, 46, (1999).

[4] B. Bidégaray, On the Cauchy problem for systems occurring in nonlinear optics, Adv. Diff. Equat., 3 (1998), 473-496.

[5] B. Bidégaray, The Cauchy problem for Schrödinger-Debye equations, Math Models Methods Appl. Sci., 10 (1998), 473-496.

[6] T. Cazenave and M. Scialom, A Schrödinger equation with time-oscillating nonlinearity, Revista Matemática Complutense, 23, (2010), 321-339.

[7] A. Corcho and F. Linares, Well-posedness for the Schrödinger-Debye equation, Contemporary Mathematics, 362 (2000), 307-315.

[8] A. Corcho and C. Matheus, Sharp bilinear estimates and well-posedness for the 1-D Schrödinger-Debye system, Differential and Integral Equations, 22 (2009), 357-391.

[9] A. Corcho, F. Oliveira and J. Drumond Silva, Local and global well-posedness for the critical Schrödinger-Debye system, Proc. Amer. Math. Soc. 141 (2013), 3485-3499.

[10] A. C. Newell and J. V. Moloney, Nonlinear Optics, Addison-Wesley, (1992).

[11] T. Tao, Nonlinear dispersive equations: Local and global analysis, CBMS, regional conference series in mathematics, American Mathematical Society, Number 106 (2006).

Instituto de Matemática - Universidade federal do Rio de Janeiro, UfRJ. Av. Athos da Silveira Ramos, P.O. Box 68530, CEP:21945-970, RJ. Brazil

E-mail address: carvajal@im.ufrj.br

Instituto de Matemática - Universidade Federal do Rio de Janeiro, UfRJ. Av. Athos da Silveira Ramos, P.O. Box 68530, CEP:21945-970, RJ. BraziL

E-mail address: pgamboa@im.ufrj.br 